\section{Benralizumab in Real Life}

Miralles López JC, Escudero Pastor AI, Carbonell Martínez A, Navarro Garrido C, Bonilla Pacheco Y, Petrik Petrik Y Hospital General Universitario Reina Sofia, Murcia, Spain

J Investig Allergol Clin Immunol 2021; Vol. 31(1): 87-88 doi: 10.18176/jiaci.0599

Key words: Severe asthma. Real life. Benralizumab.

Palabras clave: Asma grave. Vida real. Benralizumab.

\section{To the Editor:}

Benralizumab is a monoclonal antibody that binds the $\alpha$-subunit of the IL-5 receptor, leading to depletion of circulating peripheral blood eosinophils within the first 24 hours. This effect persists for at least 2 to 3 months [1,2].

Clinical trials have shown the efficacy and safety of benralizumab in patients with severe eosinophilic asthma by reducing annual disease exacerbations and improving symptoms and prebronchodilator $\mathrm{FEV}_{1}[3,4]$.

Real-life studies are important for assessing the effect of a treatment in daily clinical practice. The present report describes our experience with benralizumab under conditions of routine clinical practice.

The study population comprised 10 patients ( 6 men, 4 women; mean age, 55.9 years [range, 39-75 years]) diagnosed with severe eosinophilic bronchial asthma. Patients were treated from February 2019 to May 2020 in either Reina Sofía Hospital or Virgen de la Vega Hospital, Murcia, Spain.

We analyzed clinical characteristics, eosinophilia, total $\mathrm{IgE}$, drug tolerance and effectiveness (decreased exacerbations,
Asthma Control Test [ACT], Asthma Quality of Life Questionnaire [AQLQ], lung function $\left[\mathrm{FEV}_{1}\right.$ and $\left.\mathrm{FVC}\right]$ ), and use of oral corticosteroids. We used the Wilcoxon signed rank test for the statistical analysis; the results are reported as median (IQR).

Five patients had rhinitis and 5 nasal polyposis. Six patients were atopic. Five had never smoked and 5 were former smokers. Seven patients were obese (BMI, >30) and 2 had overweight (BMI, 25-30); the average BMI was 33.3.

Regarding severity of asthma, 5 patients were in step 5 according to the GEMA guidelines (treated with high-dose inhaled corticosteroids and long-term bronchodilators, in addition to antileukotrienes and anticholinergics), and a further 5 patients were in step 6 , requiring continuous oral corticosteroids. Seven patients had previously been treated with omalizumab and 3 with mepolizumab, although none responded to either drug. Two of the patients had received specific allergen immunotherapy. Total $\mathrm{IgE}$ levels ranged from 49 to $640 \mathrm{kU} / \mathrm{L}$, with an average of $276 \mathrm{kU} / \mathrm{L}$.

No adverse events were recorded in 9 patients, although 1 developed mild fever, which was controlled with paracetamol. This good tolerance agrees with reports on the safety of the drug [5].

Eosinophilia before therapy with benralizumab was $>600$ cells $/ \mu \mathrm{L}$ in 5 patients, between 300 and 600 cells $/ \mu \mathrm{L}$ in 2 patients, and $<300$ cells $/ \mu \mathrm{L}$ in 3 patients (the latter were receiving oral corticosteroids and/or mepolizumab treatment with previous higher eosinophil counts). The median value was 540 cells $/ \mu \mathrm{L}$ before therapy and 5 cells $/ \mu \mathrm{L}$ after (Table), with 0 eosinophils in 5 patients.

Symptoms improved with treatment in all cases, as did the number of annual exacerbations decreased, with a median of 3.50 before therapy and 0 after. It is noteworthy that 6 patients did not experience exacerbations during the year of treatment.

Table. Study Dataa

\begin{tabular}{lccc}
\hline & Before Treatment & After Treatment & Significance \\
\hline Eosinophils & $540(227.50-731.25)$ & $5(0-30)$ & $P<.005$ \\
Exacerbations/y & $3.50(2.75-6.25)$ & $0(0-1.25)$ & $P<.004$ \\
ACT & $10,50(7.75-18.00)$ & $23(17.50-24.25)$ & $P<.009$ \\
AQLQ & $2.86(1.93-4.08)$ & $5.80(4.18-6.87)$ & $P<.005$ \\
FVC, \% & $61.00(53.75-90.50)$ & $73.50(59.50-91.50)$ & $P<.124$ \\
FEV,$\%$ & $47.50(44.75-64.75)$ & $60.50(52.00-88.00)$ & $P<.036$ \\
Oral corticosteroids, mg/d & $10(8.75-20)$ & $0(0-3.75)$ & $P<.042$ \\
\hline
\end{tabular}

Abbreviations: ACT, Asthma Control Test; AQLQ, Asthma Quality of Life Questionnaire; FEV 1, forced expiratory volume in 1 second; FVC, forced vital capacity.

avalues are shown as median (IQR) 
As for disease control, 7 patients experienced a significant improvement, and there were no relevant changes in 3 patients. The median ACT value increased from 10.50 before treatment to 23 after treatment.

The AQLQ score improved in all cases, increasing from a median of 2.86 before treatment to 5.80 after treatment.

$\mathrm{FEV}_{1}, \%$ worsened in 1 patient and improved in the remainder. The median $\mathrm{FEV}_{1}, \%$ values improved from $47.50 \%$ before treatment to $60.50 \%$ after. FVC, $\%$ increased in 7 patients, decreased in 2, and remained unchanged in 1 . The median value increased from $61 \%$ to $73.50 \%$, although the difference was not statistically significant.

Finally, the median daily dose of oral corticosteroids required by 5 of the 10 patients decreased from $10 \mathrm{mg} / \mathrm{d}$ before therapy with benralizumab to $0 \mathrm{mg} / \mathrm{d}$ after. In fact, 3 patients were able to discontinue oral corticosteroids, and the other 2 lowered their dose to a quarter of the previous one.

We observed a significant improvement in clinical parameters, namely, decreased exacerbations and increased ACT and AQLQ scores. We highlight that the number of exacerbations decreased in all of the patients and that no exacerbations were recorded in 6 during the follow-up period. These results are consistent with those obtained in clinical trials with benralizumab $[3,4]$.

We also recorded an improvement in lung function test values, which was statistically significant for $\mathrm{FEV}_{1}$, although not for FVC, probably owing to the small number of patients. This improvement in lung function agrees with data from other published reports [6]. It is possible that benralizumab modulates airway remodelling pathways related to chronic eosinophil-driven inflammation.

We also recorded a significant reduction in the use of oral corticosteroids, consistent with the corticosteroid-sparing effect of benralizumab found in clinical trials [7].

In addition, benralizumab was effective in patients who did not respond to other biological treatments [8], and some of the patients who had not previously responded to other biological drugs responded well to benralizumab.

In conclusion, we found benralizumab to be a well-tolerated and effective treatment for patients with severe eosinophilic bronchial asthma, decreasing both the number of exacerbations and the intake of oral corticosteroids and improving disease control, quality of life, and lung function values.

\section{Funding}

The authors declare that no funding was received for the present study.

\section{Conflicts of Interest}

Juan Carlos Miralles López has received lecture fees from Novartis, GSK, Astra Zeneca, and Chiesi. The remaining authors declare that they have no conflicts of interest.

\section{References}

1. Ghazi A, Trikha A, Calhoun WJ. Benralizumab - a humanized $m A b$ to IL-5R $\alpha$ with enhanced antibody-dependent cellmediated cytotoxicity - a novel approach for the treatment of asthma. Expert Opin Biol Ther. 2012;12:113-8.

2. Dávila González I, Moreno Benítez F, Quirce S. Benralizumab: A New Approach for the Treatment of Severe Eosinophilic Asthma. J Invest Allergol Clin Immunol. 2019;29(2):84-93.

3. Bleecker ER, FitzGerald JM, Chanez P, Papi A, Weinstein SF, Barker $P$, et al. Efficacy and safety of benralizumab for patients with severe asthma uncontrolled with high-dosage inhaled corticosteroids and long-acting $\beta 2$-agonists (SIROCCO): a randomised, multicentre, placebo-controlled phase 3 trial. Lancet. 2016;388:2115-27.

4. FitzGerald JM, Bleecker ER, Nair P, Korn S, Ohta K, Lommatzsch $M$, et al. Benralizumab, an anti-interleukin-5 receptor $\alpha$ monoclonal antibody, as add-on treatment for patients with severe, uncontrolled, eosinophilic asthma (CALIMA): a randomised, double-blind, placebo-controlled phase 3 trial. Lancet. 2016:388:2128-41.

5. Busse WW, Bleecker ER, FitzGerald JM, Ferguson GT, Barker P, Sproule $S$, et al. Long-term safety and efficacy of benralizumab in patients with severe, uncontrolled asthma: 1-year results from the BORA phase 3 extension trial. Lancet Respir Med. 2019;7:46-59.

6. Mathur SK, Modena BD, Coumou H, Barker P, Kreindler JL, Zangrilli JG. Postbronchodilator lung function improvements with benralizumab for patients with severe asthma. Allergy. 2020;75(6):1507-10.

7. Nair P, Wenzel S, Rabe KF, Bourdin A, Lugogo NL, Kuna P, et al. Oral Glucocorticoid-Sparing Effect of Benralizumab in Severe Asthma. N Engl J Med. 2017;376:2448-58.

8. Bernal-Rubio L, de-la-Hoz Caballer B, Almonacid-Sánchez C, González-de-Olano D. Successful treatment of allergic bronchopulmonary aspergillosis with benralizumab after no response to omalizumab. J Investig Allergol Clin Immunol. 2020;30(5):378-9.

- Manuscript received May 15, 2020; accepted for publication June 8, 2020.

\section{- Juan Carlos Miralles López}

Hospital General Universitario Reina Sofía

Avda. Intendente Jorge Palacios 1

30003 Murcia, Spain

E-mail: juanc.miralles@carm.es 